\title{
One-Loop Correlators of Charged Fermionic Currents Modified by Electromagnetic Fields and Their Applications
}

\author{
Alexandra Dobrynina, ${ }^{a, b}$ llya Karabanov, ${ }^{a}$ Alexander Parkhomenko ${ }^{a, *}$ and Lubov \\ Vassilevskaya $^{c}$ \\ ${ }^{a}$ Department of Theoretical Physics, P. G. Demidov Yaroslavl State University, Sovietskaya 14, 150003 \\ Yaroslavl, Russia \\ ${ }^{b}$ II. Institute for Theoretical Physics, University of Hamburg, Luruper Chaussee 149, 22761 Hamburg, \\ Germany \\ ${ }^{c}$ Fulda University of Applied Sciences, Leipziger Straße 123, 36037 Fulda, Germany \\ E-mail: dobrynina@uniyar.ac.ru, karabanovilya@rambler.ru, \\ parkh@uniyar.ac.ru
}

\begin{abstract}
The non-diagonal correlators of tensor fermionic current with the vector and axial-vector ones are considered as examples of the two-point one-loop amplitudes modified by a constant homogeneous magnetic field. The tensor current is a fermionic part of the Pauli Lagrangian density relevant for a fermion electromagnetic interaction through its anomalous magnetic moment. Under an assumption that this interaction enters the effective QED Lagrangian, the contribution to the photon polarization operator linear in the magnetic moment is calculated. The other example where a photon interacts with a neutral pseudoscalar particle like an axion is also discussed.
\end{abstract}

\footnotetext{
*** The European Physical Society Conference on High Energy Physics (EPS-HEP2021), ***

*** 26-30 July $2021 * * *$

*** Online conference, jointly organized by Universität Hamburg and the research center DESY ***
}

\footnotetext{
${ }^{*}$ Speaker
} 


\section{Two-Point Correlators}

The Lagrangian density of the local fermion interaction has the form:

$$
\mathcal{L}_{\text {int }}(x)=\left[\bar{f}(x) \Gamma_{A} f(x)\right] J^{A}(x),
$$

where $J^{A}$ is a generalized current (like a photon, neutrino current, etc.) which includes a corresponding coupling, $\Gamma_{A}$ is any of $\gamma$-matrices from the set $\left\{1, \gamma_{5}, \gamma_{\mu}, \gamma_{\mu} \gamma_{5}, \sigma_{\mu \nu}=i\left[\gamma_{\mu}, \gamma_{\nu}\right] / 2\right\}$ or their linear combination. After generalized currents are cut, a correlation function of fermionic currents can be constructed. The two-point correlation function of general form is as follows [1]:

$$
\Pi_{A B}=\int d^{4} X \mathrm{e}^{-i(q X)} \operatorname{Sp}\left\{S_{\mathrm{F}}(-X) \Gamma_{A} S_{\mathrm{F}}(X) \Gamma_{B}\right\},
$$

where $S_{\mathrm{F}}(X)$ is the Lorentz-invariant part of an exact fermion propagator modified by an external electromagnetic field. In calculations, the Fock-Schwinger representation for fermionic propagators is used. Correlations of scalar, pseudoscalar, vector and axial-vector currents were studied [1]. Here, as examples, correlators of the tensor current with the vector and axial-vector ones are presented.

\section{Tensors in Presence of Magnetic Field}

Let us consider an external constant magnetic field with the strength $\mathbf{B}$ as the electromagnetic background. Minkowski space filled with such a field is divided into two subspaces. Its metric tensor can be written as follows: $g_{\mu \nu}=\tilde{\Lambda}_{\mu \nu}-\Lambda_{\mu \nu}$, where $\Lambda_{\mu \nu}=(\varphi \varphi)_{\mu \nu}$ and $\tilde{\Lambda}_{\mu \nu}=(\tilde{\varphi} \tilde{\varphi})_{\mu \nu}$. It includes the dimensionless tensor of the external magnetic field $\varphi_{\alpha \beta}=F_{\alpha \beta} / B$ and its dual $\tilde{\varphi}_{\alpha \beta}=\varepsilon_{\alpha \beta \rho \sigma} \varphi^{\rho \sigma} / 2$. An arbitrary four-vector $q^{\mu}=\left(q_{0}, q_{1}, q_{2}, q_{3}\right)$ can be decomposed into two orthogonal components: $q_{\mu}=\tilde{\Lambda}_{\mu \nu} q^{v}-\Lambda_{\mu \nu} q^{v}=q_{\| \mu}-q_{\perp \mu}$. Tensors $\varphi_{\alpha \beta}, \tilde{\varphi}_{\alpha \beta}, \Lambda_{\alpha \beta}=(\varphi \varphi)_{\alpha \beta}$, and the vector above allow to determine an orthogonal basis in Minkowski space: $b_{\mu}^{(1)}=(q \varphi)_{\mu}$, $b_{\mu}^{(2)}=(q \tilde{\varphi})_{\mu}, b_{\mu}^{(3)}=q^{2}(\Lambda q)_{\mu}-(q \Lambda q) q_{\mu}$, and $b_{\mu}^{(4)}=q_{\mu}$. Correlators having rank non-equal to zero, should be decomposed in this orthogonal set of vectors, in particular, a third-rank tensor $T_{\mu \nu \rho}$ can be presented as follows:

$$
T_{\mu \nu \rho}=\sum_{i, j, k=1}^{4} T_{i j k} \frac{b_{\mu}^{(i)} b_{\nu}^{(j)} b_{\rho}^{(k)}}{\left(b^{(i)} b^{(i)}\right)\left(b^{(j)} b^{(j)}\right)\left(b^{(k)} b^{(k)}\right)},
$$

where $T_{i j k}=T^{\mu \nu \rho} b_{\mu}^{(i)} b_{v}^{(j)} b_{\rho}^{(k)}$ are decomposition coefficients.

\section{Vector-Tensor Correlator}

Vector-current conservation and antisymmetry of the tensor current reduce the number of independent coefficients $Y_{i j k}^{(\mathrm{VT})}\left(q^{2}, q_{\perp}^{2}, \beta ; t, u\right)$ in the basis decomposition of the vector-tensor correlator

$$
\Pi_{i j k}^{(\mathrm{VT})}\left(q^{2}, q_{\perp}^{2}, \beta\right)=\frac{1}{4 \pi^{2}} \int_{0}^{\infty} \frac{d t}{t} \frac{\beta t}{\sin (\beta t)} \int_{0}^{1} d u Y_{i j k}^{(\mathrm{VT})}\left(q^{2}, q_{\perp}^{2}, \beta ; t, u\right) e^{-i \Omega\left(q^{2}, q_{\perp}^{2}, \beta ; t, u\right)},
$$


where $\Omega\left(q^{2}, q_{\perp}^{2}, \beta ; t, u\right)=m_{f}^{2} t-q_{\|}^{2} t\left(1-u^{2}\right) / 4+q_{\perp}^{2}[\cos (\beta t u)-\cos (\beta t)] /[2 \beta \sin (\beta t)]$ is the phase of the correlator, to 18 . Of them, four coefficients only are non-trivial $\left(Y_{i j k}^{(\mathrm{VT})}=-Y_{i k j}^{(\mathrm{VT})}\right)$ :

$$
\begin{aligned}
& Y_{114}^{(\mathrm{VT})}=-m_{f} q_{\perp}^{2} q^{2} \cos (\beta t u), \quad Y_{223}^{(\mathrm{VT})}=m_{f} q_{\perp}^{2}\left(q_{\|}^{2}\right)^{2}[\cos (\beta t)-\cos (\beta t u)], \\
& Y_{224}^{(\mathrm{VT})}=m_{f} q_{\|}^{2}\left[q_{\perp}^{2} \cos (\beta t)-q_{\|}^{2} \cos (\beta t u)\right], \quad Y_{334}^{(\mathrm{VT})}=-m_{f} q_{\perp}^{2} q_{\|}^{2}\left(q^{2}\right)^{2} \cos (\beta t u),
\end{aligned}
$$

where $q_{\|}^{2}=q^{2}+q_{\perp}^{2}$ and $\beta=e B\left|Q_{f}\right|$. All $Y_{4 j k}^{(\mathrm{VT})}$ vanish in the basis chosen.

\section{Axial-Vector-Tensor Correlator}

The correlator of the axial-vector and tensor currents is also the rank-3 tensor. Six coefficients in the basis expansion are non-trivial $\left(Y_{i j k}^{(\mathrm{AT})}=-Y_{i k j}^{(\mathrm{AT})}\right)$ :

$$
\begin{aligned}
& Y_{112}^{(\mathrm{AT})}=q_{\perp}^{2} q_{\|}^{2} u \sin (\beta t u), \quad Y_{234}^{(\mathrm{AT})}=q^{2} q_{\perp}^{2} q_{\|}^{2} \frac{\cos (\beta t) \cos (\beta t u)-1}{\sin (\beta t)}, \\
& Y_{323}^{(\mathrm{AT})}=\frac{-q_{\perp}^{2}\left(q_{\|}^{2}\right)^{2}}{\sin (\beta t)}\left\{u \sin (\beta t) \sin (\beta t u) q_{\|}^{2}-\left[2-2 \cos (\beta t) \cos (\beta t u)-\sin ^{2}(\beta t)\right] q_{\perp}^{2}\right\}, \\
& Y_{324}^{(\mathrm{AT})}=-q_{\perp}^{2} q_{\|}^{2}\left\{\left[\frac{1-\cos (\beta t) \cos (\beta t u)}{\sin (\beta t)}\right] q_{\perp}^{2}+[\cot (\beta t)[\cos (\beta t)-\cos (\beta t u)]-u \sin (\beta t u)] q_{\|}^{2}\right\}, \\
& Y_{424}^{(\mathrm{AT})}=-q_{\|}^{2}\left\{\sin (\beta t) q_{\|}^{2}-\left[\frac{2-2 \cos (\beta t) \cos (\beta t u)}{\sin (\beta t)}-u \sin (\beta t u)\right] q_{\perp}^{2}\right\} .
\end{aligned}
$$

and $Y_{423}^{(\mathrm{AT})}=Y_{324}^{(\mathrm{AT})}$.

\section{Photon Polarization Operator}

The photon polarization operator $\mathcal{P}^{\mu \nu}(q)$ is related with $\gamma \rightarrow \gamma$ transition matrix element and, consequently, with the correlator of two vector currents: $\mathcal{M}_{\gamma \rightarrow \gamma}=-i \varepsilon_{\mu}^{\prime *}(q) \mathcal{P}^{\mu \nu}(q) \varepsilon_{\nu}(q)$. The photon dispersion relations follow from the equations $q^{2}-\Pi^{(\lambda)}(q)=0$, where $\lambda=1,2,3$. $\Pi^{(\lambda)}(q)$ are eigenvalues of the photon polarization operator (the electron loop is considered only)

$$
\mathcal{P}_{\mu \nu}(q)=\sum_{\lambda=1}^{3} \frac{b_{\mu}^{(\lambda)} b_{v}^{(\lambda)}}{\left(b^{(\lambda)}\right)^{2}} \Pi^{(\lambda)}(q) .
$$

As in the vacuum, there are two physical eigenstates of $\mathcal{P}_{\mu \nu}(q)$ in the external constant homogeneous magnetic field: $\varepsilon_{\mu}^{(1)}=b_{\mu}^{(1)} / \sqrt{q_{\perp}^{2}}$ and $\varepsilon_{\mu}^{(2)}=b_{\mu}^{(2)} / \sqrt{q_{\|}^{2}}$. In the magnetic field, $\Pi^{(\lambda)}(q)$ contains both the vacuum and field-induced parts:

$$
\Pi^{(\lambda)}(q)=-i \mathcal{P}\left(q^{2}\right)-\frac{\alpha}{\pi} Y_{V V}^{(\lambda)}
$$

where $\alpha=e^{2} /(4 \pi)$ is the fine structure constant. Details about $Y_{V V}^{(\lambda)}$ can be found in [2].

Models beyond the Standard Model can give rise effectively to the Pauli Lagrangian density:

$$
\mathcal{L}_{\mathrm{AMM}}(x)=-\frac{\mu_{B} a_{e}}{2}\left[\bar{\psi}(x) \sigma_{\mu \nu} \psi(x)\right] F^{\mu v}(x),
$$


where $\mu_{B}=e /\left(2 m_{e}\right)$ is the Bohr magneton and $a_{e}$ is the electron anomalous magnetic moment (AMM). Due to $\mathcal{L}_{\mathrm{AMM}}$, an additional terms in (7) are produced. The contribution linear in the electron $\mathrm{AMM}$ is related with the correlator of the vector and tensor currents, $\Pi_{\mu \nu \rho}^{(\mathrm{VT})}$. The fieldinduced part of $\Pi^{(\lambda)}(q)$ is modified by the additional term $\alpha a_{e} Y_{V T}^{(\lambda)} / \pi$, where

$$
\begin{aligned}
& Y_{V T}^{(\lambda)}=\int_{0}^{\infty} \frac{d t}{t} \int_{0}^{1} d u\left\{\frac{\beta t}{\sin (\beta t)} y_{V T}^{(\lambda)} e^{-i \Omega}-q^{2} e^{-i \Omega_{0}}\right\}, \quad \Omega_{0}=m_{f}^{2} t-q^{2} t\left(1-u^{2}\right) / 4 . \\
& y_{V T}^{(1)}=y_{V T}^{(3)}=q^{2} \cos (\beta t u), \quad y_{V T}^{(2)}=q_{\|}^{2} \cos (\beta t u)-q_{\perp}^{2} \cos (\beta t),
\end{aligned}
$$

Notations are taken from [2]. The part independent on the field is subtracted. For the electron, $a_{e} \sim \alpha$ and the AMM correction is small.

\section{Axion-Photon Mixing}

Axion-photon mixing is arising from a local interaction of an axion with two photons when bosons are propagating in an external magnetic background [3]. The local axial-vector interaction of an axion with fermions:

$$
\mathcal{L}_{a f}(x)=-\frac{C_{a}}{2 f_{a}}\left[\bar{\psi}(x) \gamma_{\mu} \gamma_{5} \psi(x)\right] \partial^{\mu} a(x),
$$

modifies this mixing. An existence of the Pauli Lagrangian $\mathcal{L}_{\mathrm{AMM}}(x)$ gives an extra term into the $a \rightarrow \gamma$ transition matrix element which is related with the correlator of the axial-vector and tensor currents, $\Pi_{\mu \nu \rho}^{(\mathrm{AT})}$. The one with $Y_{424}^{(\mathrm{AT})}$ contributes into the mixing of axions with photons of the polarization $\epsilon_{\mu}=b_{\mu}^{(2)}$. More details are presented in a forthcoming publication.

\section{Conclusions}

Two-point correlators in the presence of the constant homogeneous external magnetic field are considered. Study of correlators of the tensor fermionic current with the others allows to investigate effects of the fermion AMM in the one-loop approximation. The field-induced contributions to the photon polarization operator and axion-photon mixing are discussed.

\section{Acknowledgements}

This work is supported by the RSF (Grant No. 18-72-10070). A. D. acknowledges the support by the Alexander von Humboldt Foundation.

\section{References}

[1] M. Y. Borovkov, A. V. Kuznetsov and N. V. Mikheev, Phys. Atom. Nucl. 62 (1999) 1601.

[2] A. Kuznetsov and N. Mikheev, Electroweak Processes in External Active Media, SpringerVerlag, New York 2013.

[3] G. G. Raffelt, Stars as Laboratories for Fundamental Physics: The astrophysics of neutrinos, axions, and other weakly interacting particles, University of Chicago Press, Chicago \& London 1996. 